\title{
Headache associated with sexual activity
}

Frese et al. analyzed the clinical features of this headache in 51 patients. They found a male preponderance. Most patients have an explosive pain at orgasm. Attacks were not related to any particular sexual habit. There was a high comorbidity with other primary headache disorders.

see page 796

Commentary by David W. Dodick, MD

Headache associated with sexual activity ... gets your attention

There are few headaches that grab the attention of patients and physicians more than those associated with sexual activity. Apart from their noteworthy and peculiar intrusiveness, these headaches are most often apoplectic in onset, occur at the moment of orgasm, and as such, closely resemble the thunderclap headache sometimes associated with potentially catastrophic intracranial diseases such as subarachnoid hemorrhage (SAH). The physician's apprehension is justifiably heightened by several facts: sudden severe headache is the most common symptom of a $\mathrm{SAH}$, the sole manifestation in about $12 \%$ of cases, and precedes a catastrophic rebleed in up to $50 \%$ of patients. ${ }^{1,2}$ Moreover, subarachnoid hemorrhage occurs during sexual activity in up to $11 \%$ of cases. Headache associated with sexual activity (HSA) itself often occurs repeatedly and can be quite debilitating. Therefore, any attempt to elucidate the distinguishing clinical features and underlying mechanism of this disorder is welcome.

The recently revised diagnostic criteria of the International Headache Society distinguish between two types of HSA: preorgasmic (previously known as HAS type I) and orgasmic headache (previously known as HAS type II). The main distinguishing feature is the presence or absence of a headache that gradually intensifies until orgasm in preorgasmic compared to the sudden onset of a headache simultaneous with orgasm in the latter. There was insufficient evidence to provide more explicit details regarding their duration, associated symptoms, and other clinical features that may prove useful to clinicians in determining the timing and extent of a diagnostic evaluation. In light of this, Frese et al. have conducted a careful interview study of 51 patients with HSA confirming previously reported features and refining the clinical spectrum of this disorder. Generally, these patients are young (mean age 39 years) males (3:1) with orgasmic headache (82\%) that is bilateral, and diffuse or occipital (76\%). Severe headaches usually last 30 minutes and rarely longer than 12 hours, although mild pain usually persists for up to 3 days after the ictus. Importantly, almost $50 \%$ of patients can terminate the headache by either stopping or assuming a more passive role during sexual activity. Focal neurologic symptoms or signs are not seen, although dizziness and vomiting may be present. Interestingly, benign exertional headache, which has a popu- lation prevalence of only $1 \%$, occurs in one-third of these patients. This likely reflects a shared mechanism, postulated to be a result of a transient elevation in intracranial pressure or impaired cerebrovascular autoregulation.

These features may be helpful to clinicians in practice. However, because $12 \%$ of patients with SAH present only with headache that may be indistinguishable from orgasmic headache, the only distinctive feature permitting a clinical diagnosis of HSA is a history of multiple prior stereotyped and selflimited episodes of orgasmic or preorgasmic headache. Fortunately, once the diagnosis is made, patients can be reassured. Moreover, they can often be treated effectively with indomethacin. ${ }^{3}$

\section{References}

1. Edlow JA, Caplan LR. Avoiding pitfalls in the diagnosis of subarachnoid hemorrhage. N Engl J Med 2000;342: 29-36.

2. Weir B. Headaches from aneurysms. Cephalalgia 1994;14:79-87.

3. Pascual J, Iglesias F, Oterino A, Vazquez-Barquero A, Bercian J. Cough, exertional, and sexual headache: an analysis of 72 benign and symptomatic cases. Neurology 1996; 46:1520-1524.

see page 796 


\section{AAN treatment recommendation for Guillain-Barré syndrome}

Hughes et al. used Cochrane systematic reviews of randomized controlled trials as the basis for their conclusions concerning Guillain-Barré syndrome. They recommend plasma exchange or IV immunoglobulin as of equivalent efficacy in adults with early severe disease. Their value in mild disease and in children is not known. IV immunoglobulin is more convenient and somewhat safer. Corticosteroids are not efficacious and not recommended.

see page 736

\section{ALS in Gulf War veterans?}

Veterans at increased risk

Horner et al. conducted a nationwide case ascertainment study to determine if 1991 Gulf War veterans were at increased risk of ALS. Over the 10-year follow-up period, 107 confirmed cases of ALS were identified among the 2.4 million military personnel on active duty during the Gulf War, yielding an almost twofold higher risk among those who had been deployed to the Gulf Region. The reason for the increase remains unknown.

see page 742

Excess incidence in young veterans

In a study of the first ALS cases in Gulf War veterans, Robert W. Haley reports that observed incidence below age 45 was 2.3 times expected in 1995 through 1998 and 3.2 times expected in 1998. Rising incidence and preceding Gulf War illness in 65\% suggest a war-related environmental trigger.

see page 750

Even if a twofold excess risk is correct, the baseline rarity of ALS means at worst an increase

in the chances of getting ALS to 1 in 150,000 .
In the accompanying editorial, $\mathrm{Mi}$ chael $R$. Rose notes that formal recognition of $A L S$ as a Gulf Warrelated illness by the Veterans $A d-$ ministration of the United States was made on humane and political grounds but has been taken as quasi-scientific validation of the claim. These two articles allow readers to judge for themselves the claims of an excess risk of developing ALS in Gulf War veterans. Rose concludes that the excess risk is not convincing, especially given the small number of ALS cases. In the time since these studies were performed, mortality from ALS in Gulf War veterans has not risen as one would have expected. The age distribution of incidence of ALS should shift toward the younger age group as would be expected if a premature triggering agent is operative.

\section{Intracranial ictal onset zone of lateral TLE}

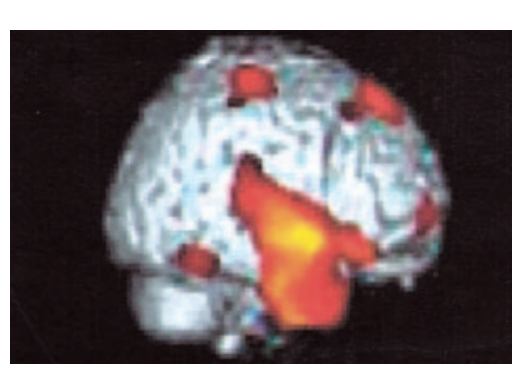

Lee et al. showed that scalp video-EEG recordings could be falsely localizing in patients with nonlesional lateral temporal lobe seizures. One-third of their patients had an extratemporal ictal onset zone (frontal, parietal, or occipital) when subsequent in-

Hetabolic (epileptic focu visualized by FDG-PET and statistical parametric mapping.

vasive monitoring was performed with grids or strip electrodes. Their work suggests that careful placement of intracranial electrodes on the temporal lobe and the adjacent areas is required for these patients so as to determine if surgery is a potential therapy.

see page 757

In an accompanying editorial, Fred Andermann focuses on the diagnostic difficulties presented by these patients. He stresses the importance of taking into account seizure behavior and the presence of extratemporal lesions and even prior EEG results as a way of avoiding the incorrect diagnosis of temporal lobe seizures based on scalp monitoring. Even in nonlesional patients, there may be subtle and unapparent structural abnormalities on MRI, PET, or SPECT imaging, such as cortical dysplasia. In extratemporal sites, seizures could be generated, but these may be expressed primarily through the temporal lobe, resulting in falsely localizing scalp EEG records. These considerations support Lee et al.'s conclusion that with a high level of suspicion, extratemporal foci can be identified with invasive monitoring. Surgery-with potential for a good outcome-can then be considered.

see page 732

\section{Mania after temporal lobectomy}

Carran et al. compared patients with new onset mania following anterior temporal lobectomy (ATL) with control and postoperative depression groups. Predictors of mania included preoperative diagnostic tests lateralizing to the nonsurgical hemisphere, in particular bilateral temporal EEG abnormalities, and right ATL.

see page 770 


\section{Productive infection of neurons by JC virus}

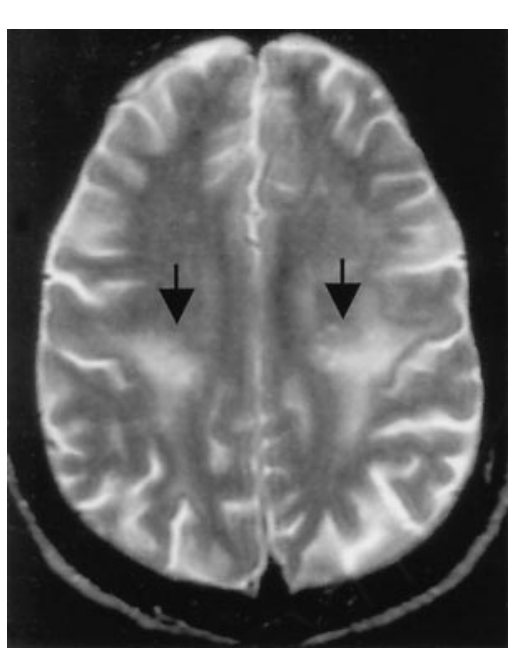

PML lesions in frontal lobes: subcortical hyperintensities (arrows).

Du Pasquier et al. studied the brain of an HIV+ patient who died of progressive multifocal leukoencephalopathy caused by the polyomavirus JC. They demonstrated that, in addition to oligodendrocytes, this virus was able to productively infect granule cell neurons of the cerebellum.

see page 775

The accompanying editorial by Ken Tyler reviews the pathogenesis of JC virus infection of the CNS, and how infection can lead to development of progressive multifocal leukoencephalopathy in immunocompromised hosts. The Du Pasquier et al. study extends the known tropism of $\mathrm{JV}$ virus to encompass cerebellar granular neurons. Understanding and targeting viral tropism and replication has been essential for all current antiviral therapies and may foster design of effective antiviral drugs for $P M L$

see page 734

\section{Cognitive functioning in the menopausal transition}

In a population-based study of the menopausal transition, yearly assessments over 5 years for 803 women included simple assessments of working memory (Digit Span Backward) and perceptual speed (Symbol Digit Modalities Test). Meyer et al. found no decline associated with progression through the menopausal transition.

see page 801

\section{Retinopathy and cerebral atrophy}

In a study of 1684 middle-aged people, Wong et al. showed that those with retinopathy, as detected from retinal photographs, were more likely to have cerebral atrophic changes on MRI, independent of vascular risk factors. This suggests that microvascular processes may influence the development of cerebral atrophy.

see page 806

\section{Late onset presentation of hereditary myasthenia}

Burke et al. describe distinct early and late-onset phenotypes for myasthenia due to rapsyn mutations. The patients with late-onset presentation had previously been misdiagnosed with seronegative MG.

see page 826

\section{Prognosis after negative biopsy for CNS angiitis}

Alreshaid and Powers analyzed 25 patients with a nondiagnostic brain biopsy for clinically suspected CNS angiitis. Good outcome at 1 year was seen in 6 out of the 10 patients treated with immunosuppressive therapy, as compared to 8 out of the 15 not treated.

see page 831

see page 783

\section{Neurologist vs non-neurologist stroke care}

Goldstein et al. analyzed the care of 7775 patients with stroke admitted to one of nine VA hospitals based on physician specialty (neurologist care, $\mathrm{n}=614$; nonneurologist, $\mathrm{n}=161$ ). Neurologists obtained more tests, but their patients were less likely to die or be dependent $(\mathrm{OR}=0.63, \mathrm{p}=0.025)$ at the time of discharge.

see page 792

\section{Pergolide and heart valvular disease}

Van Camp et al. report two pergolide-treated patients with Parkinson disease who developed valvular heart disease. The valvular lesions of one patient were typical for ergot- or carcinoid-induced valvlopathy. The echocardiographic data of another eight patients treated with high doses of pergolide further supported a role of pergolide in restrictive valvular disease.

see page 859 


\section{Neurology}

September 23 Highlights

Neurology 2003;61;727-729

DOI 10.1212/WNL.61.6.727

This information is current as of September 22, 2003

Updated Information \&

Services

References

Permissions \& Licensing

Reprints including high resolution figures, can be found at: http://n.neurology.org/content/61/6/727.full

This article cites 3 articles, 1 of which you can access for free at: http://n.neurology.org/content/61/6/727.full\#ref-list-1

Information about reproducing this article in parts (figures,tables) or in its entirety can be found online at:

http://www.neurology.org/about/about_the_journal\#permissions

Information about ordering reprints can be found online:

http://n.neurology.org/subscribers/advertise

Neurology ${ }^{\circledR}$ is the official journal of the American Academy of Neurology. Published continuously since 1951, it is now a weekly with 48 issues per year. Copyright . All rights reserved. Print ISSN: 0028-3878. Online ISSN: 1526-632X.

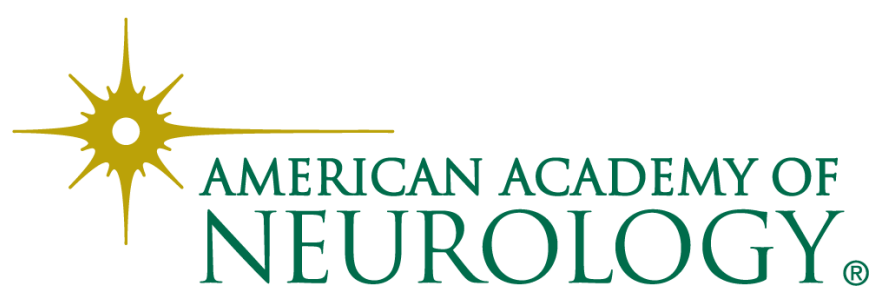

